Mathematics

Feb 2015

\title{
Moving Randomly in Three Dimensions
}

Making probabilistic inferences about spatial locations of a vector at certain points of time is important in both theoretical and applied mathematics. For his thesis work as part of Ryerson's MSc Program in Applied Mathematics, Xianzhang Wen undertook studies in this area. His focus was on finding analytical solutions for multi-dimensional partial differential equations required to price financial products.

In collaboration with his supervisors, Dr. Marcos Escobar and Dr. Sebastian Ferrando, Xianzhang recently published findings from this research in two separate articles. The first, which appeared in Stochastics: An International Journal of Probability and Stochastic Processes, deals with the mathematical problem; the second, published in the International Journal of Financial Markets and Derivatives, applies the results to financial mathematics.

\section{Probabilistic Dependency}

Xianzhang's investigations involved studying a three-dimensional random vector, each component of which moves stochastically (i.e., its time evolution is unpredictable, but the vector's coordinates are correlated). As an example, one can think of the stock-chart values of three different companies in the same industrial sector. It is impossible to predict their value tomorrow; however, the charts will have a probabilistic dependency.

That's where Xianzhang's research stepped in, by attempting to answer this question: "What is the probability that at least one of the components of the three-dimensional vector will fall below a certain level?" In his thesis, Xianzhang cast light on such concerns for certain correlation structures. The core of his research required partitioning a three-dimensional sphere via spherical triangles for different specifications (see figures 1 and 2); this was a key technique for establishing his solutions.

Dr. Ferrando emphasizes the importance of Xianzhang's work: "This is the first work to provide explicit, exact solutions - as opposed to approximations - for the three-dimensional case for a limited set of correlation structures."

\section{Strong Support}

Since completing his MSc, Xianzhang has become a market risk and model development analyst at TD CanadaTrust. Looking back on his studies at Ryerson, he notes that "the most enjoyable aspect was the good relationship I had with my supervisors. I benefited from weekly discussions with Drs. Escobar and Ferrando, and they provided many critical and useful suggestions when I encountered difficulties during my research."

\section{Funding}

Xianzhang's research was supported by an Ontario Graduate Scholarship and NSERC. 